\title{
Radiation From a Microstrip Amplifier
}

\author{
Tzyy-Sheng Horng, Member, IEEE, and Sung-Mao Wu
}

\begin{abstract}
A full-wave method is presented to investigate radiation from a microstrip amplifier. The spectral-domain dyadic Green's function, which takes into account both radiation and surface waves, is used to formulate an integral equation. The method of moments is then employed to find the current densities in microstrips and, subsequently, the scattering parameters of the amplifier. The radiated space and surface waves that are launched from the amplifier can be further expressed in terms of the dyadic Green's function and current densities. To verify the numerical results of scattering parameters and far-field radiation patterns, a UHF-band microstrip amplifier matching with single stubs has been implemented and measured. The comparison between simulation and measurement shows excellent agreement.
\end{abstract}

Index Terms-Full-wave method, microstrip amplifier, radiation loss, spectral-domain approach, surface-wave loss.

\section{INTRODUCTION}

$\mathbf{I}$ $\mathrm{N}$ THE PAST, space- and spectral-domain approaches applied to the electric-field integral equations (EFIEs) [1]-[5] and mixed-potential integral equations (MPIEs) [6]-[8] have been used extensively to characterize the microstrip passive components. For analysis with an active component, one can import the full-wave scattering-parameter results as a black box and cascade them with an active device model using a conventional microwave computer-aided design (CAD) tool. However, the RF parameters that can be evaluated are not extended to radiation and surface-wave losses. Recently, new techniques based on the finite-difference time-domain (FDTD) method [9]-[11] have been reported in simulating microstrip active circuits. Although showing great potential in predicting the electromagnetic interference, these new techniques still have difficulties in distinguishing between radiation and surface waves.

In this paper, the spectral-domain approach applied to the EFIE is formulated to investigate a microstrip amplifier that comprises arbitrarily shaped junctions, vias, and a GaAs MESFET. The three-dimensional metallization structure requires a current segmentation scheme in the moment-method procedure. It was found that, for microstrip structures, the use of rectangular basis functions, as proposed in [2], is quite efficient numerically. After the current densities in the microstrip traces have been found, the scattering parameters can be extracted. The spectral-domain EFIE formulation can be interpreted by superposition of plane-wave functions. One can

Manuscript received January 18, 1999. This work was supported by the National Science Council, Taiwan, R.O.C., under Grant NSC-89-2213-E-110-021.

The authors are with the Department of Electrical Engineering, National Sun Yat-Sen University, Taiwan 804, R.O.C. (e-mail: jason@ee.nsysu.edu.tw).

Publisher Item Identifier 10.1109/TMTT.2002.801359.

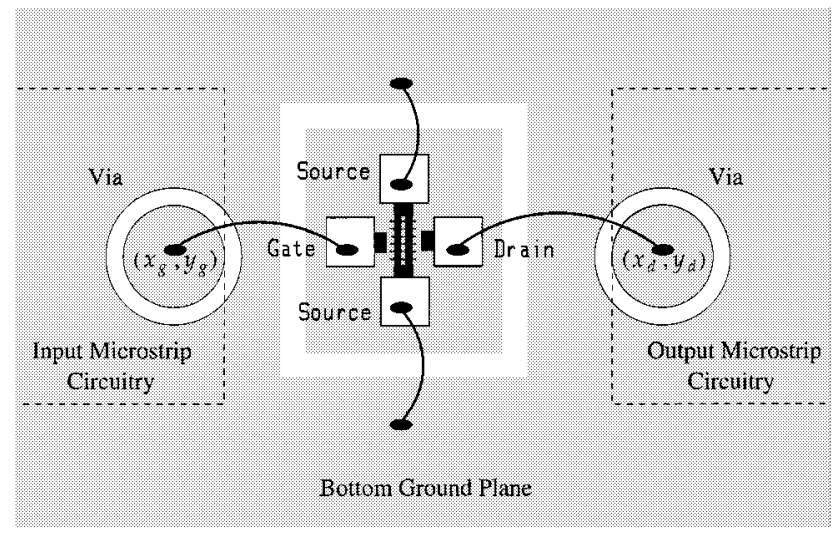

Fig. 1. GaAs MESFET die attached to the bottom ground plane of the substrate.

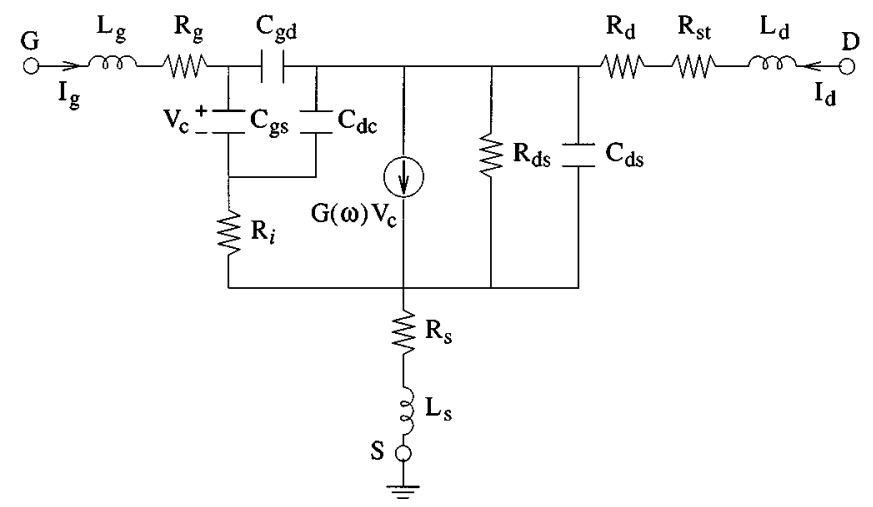

Fig. 2. Small-signal equivalent circuit of a GaAs MESFET.

differentiate between radiation and surface waves by examining their plane-wave spectra [12], [13]. The losses due to radiation and surface waves are finally evaluated through a rigorous Poynting vector analysis.

\section{THEORY}

\section{A. Impedance Parameters for the GaAs MESFET Small-Signal Model}

Fig. 1 shows a typical common source configuration for a GaAs MESFET. For this device to function as an amplifier, proper bias conditions need to be established. This determines the values of intrinsic network parameters in the small-signal equivalent circuit, as shown in Fig. 2. The extrinsic parameters include the source, gate, and drain inductances due to bond wires and a stability resistance. By referring to [13] and [14], the two-port impedance parameters for such an equivalent cir- 
cuit are derived as

$$
\begin{aligned}
& Z_{12}(\omega)=j \omega L_{s}+R_{s}+\frac{T_{s}(\omega)}{T_{n}(\omega)} \\
& Z_{11}(\omega)=j \omega L_{g}+\left(R_{g}+R_{i}\right)+\frac{1}{j \omega C_{\mathrm{gs}}}-\frac{T_{g}(\omega)}{T_{n}(\omega)}+Z_{12}(\omega) \\
& Z_{21}(\omega)=\frac{T_{e}(\omega)}{T_{n}(\omega)}+Z_{12}(\omega) \\
& Z_{22}(\omega)=j \omega L_{d}+R_{d}+R_{\mathrm{st}}+Z_{\mathrm{ds}}(\omega)-\frac{T_{d}(\omega)}{T_{n}(\omega)}+Z_{12}(\omega)
\end{aligned}
$$

where

$$
\begin{aligned}
& Z_{\mathrm{ds}}(\omega)=\frac{R_{\mathrm{ds}}}{1+j \omega R_{\mathrm{ds}} C_{\mathrm{ds}}} \\
& T_{g}(\omega)=\left(R_{i}^{2}+R_{i} Z_{\mathrm{ds}}(\omega)\right)\left(\frac{1}{C_{\mathrm{gd}}}+\frac{1}{C_{\mathrm{dc}}}+\frac{1}{C_{\mathrm{gs}}}\right) \\
& +\frac{G(\omega) Z_{\mathrm{ds}}(\omega) R_{i}}{j \omega C_{\mathrm{gs}} C_{\mathrm{gd}}}+\frac{R_{i}+Z_{\mathrm{ds}}(\omega)}{j \omega C_{\mathrm{gs}}^{2}} \\
& +\frac{1}{j \omega C_{\mathrm{gs}} C_{\mathrm{dc}}}\left(2 R_{i}+Z_{\mathrm{ds}}(\omega)+G(\omega) Z_{\mathrm{ds}}(\omega) R_{i}\right. \\
& \left.+\frac{1+G(\omega) Z_{\mathrm{ds}}(\omega)}{j \omega C_{\mathrm{gs}}}\right) \\
& T_{d}(\omega)=\left(\frac{1}{C_{\mathrm{gd}}}+\frac{1}{C_{\mathrm{dc}}}+\frac{1}{C_{\mathrm{gs}}}\right)\left(Z_{\mathrm{ds}}^{2}(\omega)+Z_{\mathrm{ds}}(\omega) R_{i}\right) \\
& +\frac{Z_{\mathrm{ds}}(\omega)+G(\omega) Z_{\mathrm{ds}}^{2}(\omega)}{j \omega C_{\mathrm{dc}} C_{\mathrm{gs}}} \\
& T_{s}(\omega)=Z_{\mathrm{ds}}(\omega) R_{i}\left(\frac{1}{C_{\mathrm{gd}}}+\frac{1}{C_{\mathrm{dc}}}+\frac{1}{C_{\mathrm{gs}}}\right)+\frac{Z_{\mathrm{ds}}(\omega)}{j \omega C_{\mathrm{dc}} C_{\mathrm{gs}}} \\
& T_{n}(\omega)=\left(\frac{1}{C_{\mathrm{gd}}}+\frac{1}{C_{\mathrm{dc}}}+\frac{1}{C_{\mathrm{gs}}}\right)\left(R_{i}+Z_{\mathrm{ds}}(\omega)\right) \\
& +\frac{1}{C_{\mathrm{dc}}}\left(\frac{1+G(\omega) Z_{\mathrm{ds}}(\omega)}{j \omega C_{\mathrm{gs}}}+\frac{1}{j \omega C_{\mathrm{gd}}}\right) \\
& T_{e}(\omega)=\frac{G(\omega) Z_{\mathrm{ds}}(\omega)}{\omega^{2} C_{\mathrm{gs}} C_{\mathrm{dc}} C_{\mathrm{gd}}}-\frac{G(\omega) Z_{\mathrm{ds}}(\omega) R_{i}}{j \omega C_{\mathrm{gs}} C_{\mathrm{gd}}} \\
& G(\omega)=g_{m} e^{-j \omega \tau} \text {. }
\end{aligned}
$$

\section{B. Spectral-Domain Analysis of a Microstrip Amplifier}

Fig. 3 shows the design of the input and output matching circuitry implemented on the top of the substrate. The GaAs FET is placed on the bottom ground plane, as shown in Fig. 1. Its gate and drain pads are wire bonded to the vias, which are connected to the microstrip lines. In the spectral-domain approach, the space-domain electric field can be expressed as the inverse Fourier transform of the vector product of spectral-domain dyadic Green's function and the Fourier transform of the current densities. Assuming that the microstrip lines are perfect

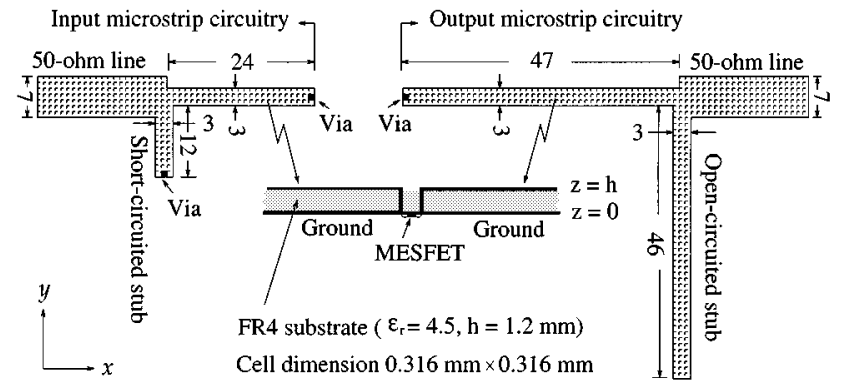

Fig. 3. Layout segmentation for the matching circuitry of a microstrip amplifier implemented on the top of the FR4 substrate.

conductors, the EFIE is generally written as

$$
\begin{aligned}
\frac{1}{4 \pi^{2}} & \int_{-\infty}^{\infty} \int_{-\infty}^{\infty} \int_{z^{\prime}} \overline{\overline{\tilde{G}}}\left(k_{x}, k_{y}, z, z^{\prime}\right) \cdot \overrightarrow{\tilde{J}}\left(k_{x}, k_{y}, z^{\prime}\right) \\
& \cdot e^{-j k_{x} x} e^{-j k_{y} y} d z^{\prime} d k_{x} d k_{y} \\
= & \vec{E}_{\mathrm{exc}}(x, y, z), \quad \text { for } x, y, z \text { on the conductor }
\end{aligned}
$$

where $\overline{\overline{\widetilde{G}}}$ and $\overrightarrow{\widetilde{J}}$ denote the spectral-domain dyadic Green's function and Fourier transforms of the current densities, respectively. $\vec{E}_{\text {exc }}$ is the impressed field due to the voltage excitation. When the method of moments is used to solve the above equation, the current densities in a three-dimensional metallization structure, as shown in Fig. 3, are discretized into a number of rectangular cells. Under this scheme, the surface current density on the microstrip lines is expanded by a number of rooftop functions, whereas the vertically directed volume current density in a via is approximated using a rectangular post [2], [5]. After transforming into the spectral domain, the microstrip current densities can be expressed as

$$
\begin{aligned}
\overrightarrow{\widetilde{J}}\left(k_{x}, k_{y}, z^{\prime}\right)= & \sum_{m=1}^{M} \frac{I_{x}^{m}}{d_{y}} \tilde{j}_{x}^{m}\left(k_{x}, k_{y}\right) \delta\left(z^{\prime}-h\right) \\
& +\sum_{n=1}^{N} \frac{I_{y}^{n}}{d_{x}} \tilde{j}_{y}^{n}\left(k_{x}, k_{y}\right) \delta\left(z^{\prime}-h\right) \\
& +\sum_{k=1}^{K} \frac{I_{z}^{k}}{d_{x} d_{y}} \tilde{j}_{z}^{k}\left(k_{x}, k_{y}\right) P\left(\frac{z^{\prime}-0.5 h}{h}\right)
\end{aligned}
$$

where

$$
\tilde{j}_{x}^{m}\left(k_{x}, k_{y}\right)=\frac{2-2 \cos \left(k_{x} d_{x}\right)}{k_{x}^{2} d_{x}} \frac{\sin \left(k_{y} d_{y} / 2\right)}{k_{y} / 2} e^{j k_{x} x_{m}} e^{j k_{y} y_{m}}
$$

$$
\begin{aligned}
\tilde{j}_{y}^{n}\left(k_{x}, k_{y}\right) & =\frac{\sin \left(k_{x} d_{x} / 2\right)}{k_{x} / 2} \frac{2-2 \cos \left(k_{y} d_{y}\right)}{k_{y}^{2} d_{y}} e^{j k_{x} x_{n}} e^{j k_{y} y_{n}} \\
\tilde{j}_{z}^{k}\left(k_{x}, k_{y}\right) & =\frac{\sin \left(k_{x} d_{x} / 2\right)}{k_{x} / 2} \frac{\sin \left(k_{y} d_{y} / 2\right)}{k_{y} / 2} e^{j k_{x} x_{k}} e^{j k_{y} y_{k}} \\
P(u) & = \begin{cases}1, & |u| \leq 1 / 2 \\
0, & |u|>1 / 2\end{cases}
\end{aligned}
$$


By substituting (13) into (12), it is found that the integration with respect to $z^{\prime}$ can be evaluated in closed form. This results in the spectral-domain expression of the electric field given as

$$
\begin{aligned}
\overrightarrow{\widetilde{E}}\left(k_{x}, k_{y}, z\right) & \\
= & \int_{0}^{h} \overline{\overline{\tilde{G}}}\left(k_{x}, k_{y}, z, z^{\prime}\right) \cdot \overrightarrow{\tilde{J}}\left(k_{x}, k_{y}, z^{\prime}\right) d z^{\prime} \\
= & {\left[\begin{array}{l}
\tilde{G}_{x x}\left(k_{x}, k_{y}, z\right) \\
\tilde{G}_{y x}\left(k_{x}, k_{y}, z\right) \\
\tilde{G}_{z x}\left(k_{x}, k_{y}, z\right)
\end{array}\right] \sum_{m=1}^{M} \frac{I_{x}^{m}}{d_{y}} \tilde{j}_{x}^{m}\left(k_{x}, k_{y}\right) } \\
& +\left[\begin{array}{c}
\tilde{G}_{x y}\left(k_{x}, k_{y}, z\right) \\
\tilde{G}_{y y}\left(k_{x}, k_{y}, z\right) \\
\tilde{G}_{z y}\left(k_{x}, k_{y}, z\right)
\end{array}\right] \sum_{n=1}^{N} \frac{I_{y}^{n}}{d_{x}} \tilde{j}_{y}^{n}\left(k_{x}, k_{y}\right) \\
& +\left[\begin{array}{l}
\tilde{G}_{x z}\left(k_{x}, k_{y}, z\right) \\
\tilde{G}_{y z}\left(k_{x}, k_{y}, z\right) \\
\tilde{G}_{z z}\left(k_{x}, k_{y}, z\right)
\end{array}\right] \sum_{k=1}^{K} \frac{I_{z}^{k}}{d_{x} d_{y}} \tilde{j}_{z}^{k}\left(k_{x}, k_{y}\right) .
\end{aligned}
$$

All the components of the spectral-domain dyadic Green's functions in (18) can be found in [4]. For the excitation, an impressed electric field in the $x$-direction is generated by a voltage source across an infinitesimal gap near the edge of input microstrip line. An $x$-directed semi-infinite traveling-wave function is also used for current expansion in the output microstrip line to exclude the effects of the reflected currents [1]. To deal with the active elements, in Fig. 1, one can assume that the via connected to the gate pad has a voltage potential $I_{g} Z_{11}+I_{d} Z_{12}$ with respect to ground, whereas the via connected to the drain pad has a voltage potential equal to $I_{d} Z_{22}+I_{g} Z_{21}$. Thus, the electric field for excitation can be approximated as

$$
\begin{aligned}
\vec{E}_{\mathrm{exc}}(x, y, z) \approx & V_{0} \delta\left(x-x_{s}\right) \hat{x}+\left[I_{g} Z_{11}(\omega)+I_{d} Z_{12}(\omega)\right] \\
& \cdot P\left(\frac{x-x_{g}}{d_{x}}\right) P\left(\frac{y-y_{g}}{d_{y}}\right) \delta(z) \hat{z} \\
& +\left[I_{d} Z_{22}(\omega)+I_{g} Z_{21}(\omega)\right] \\
& \cdot P\left(\frac{x-x_{d}}{d_{x}}\right) P\left(\frac{y-y_{d}}{d_{y}}\right) \delta(z) \hat{z}
\end{aligned}
$$

Substitute (18) and (19) into (12) and apply the method of moments to convert the integral equations into a matrix form given as

$$
\begin{aligned}
& {\left[\begin{array}{lll}
{\left[Z_{j_{x} j_{x}}\right]_{M \times M}} & {\left[Z_{j_{y} j_{x}}\right]_{N \times M}} & {\left[Z_{j_{z} j_{x}}\right]_{K \times M}} \\
{\left[Z_{j_{x} j_{y}}\right]_{M \times N}} & {\left[Z_{j_{y} j_{y}}\right]_{N \times N}} & {\left[Z_{j_{z} j_{y}}\right]_{K \times N}} \\
{\left[Z_{j_{x} j_{z}}\right]_{M \times K}} & {\left[Z_{j_{y} j_{z}}\right]_{N \times K}} & {\left[Z_{j_{z} j_{z}}\right]_{K \times K}}
\end{array}\right]\left[\begin{array}{l}
{\left[I_{x}\right]_{M}} \\
{\left[I_{y}\right]_{N}} \\
{\left[I_{z}\right]_{K}}
\end{array}\right]} \\
& =\left[\begin{array}{l}
{\left[V_{x}\right]_{M}} \\
{\left[V_{y}\right]_{N}} \\
{\left[V_{z}\right]_{K}}
\end{array}\right] \text {. }
\end{aligned}
$$

Each submatrix on the left-hand side of (20) represents a set of mutual impedances between expansion functions and testing functions. For the excitation submatrices on the right-hand side, since a delta function is assumed for each component in (19), integrating over the testing functions yields nonzero elements for $\left[V_{x}\right]$ and $\left[V_{z}\right]$. It is noted that the submatrix $\left[V_{z}\right]$ contains the transistor current coefficients $I_{g}, I_{d}$ to be determined and can be moved to the left-hand side before the matrix inversion is performed to compute all the unknown current coefficients.

\section{Evaluation of Radiation and Surface-Wave Losses}

Once the dyadic Green's function and current densities have been found, in principle, the electromagnetic fields in all space can be determined. By referring to (18), the scattered electric field in the space domain due to the microstrip current densities is expressed as

$$
\begin{aligned}
\vec{E}_{s}(x, y, z)=\frac{1}{4 \pi^{2}} \int_{-\infty}^{\infty} \int_{-\infty}^{\infty} \overrightarrow{\tilde{E}}\left(k_{x}, k_{y}, z\right) \\
\cdot e^{-j k_{x} x} e^{-j k_{y} y} d k_{x} d k_{y} .
\end{aligned}
$$

The above expression includes both radiated space and surface waves. The radiated space waves propagate upward from the top of the substrate into free space. Therefore, for the variation in the vertical direction, a traveling-wave function $e^{-j k_{z 0} z}$ is assumed and factored out. Note that the wavenumber $k_{z 0}$ has to be positive real. Thus,

$$
k_{z 0}^{2}=k_{0}^{2}-k_{x}^{2}-k_{y}^{2}>0 .
$$

The electromagnetic fields of radiated space waves in the freespace region $(z>h)$ can be then characterized by the forms

$$
\begin{aligned}
\vec{E}_{r}(x, y, z)= & \frac{1}{4 \pi^{2}} \iint_{k_{x}^{2}+\vec{k}_{y}^{2}<k_{0}^{2}} \overrightarrow{\vec{E}}_{r}\left(k_{x}, k_{y}\right) e^{-j k_{z 0} z} \\
& \cdot e^{-j k_{x} x} e^{-j k_{y} y} d k_{x} d k_{y} \\
\vec{H}_{r}(x, y, z)= & \frac{j \nabla \times \vec{E}_{r}(x, y, z)}{\omega \mu_{0}} \\
= & \frac{1}{4 \pi^{2} \omega \mu_{0}} \iint_{k_{x}^{2}+k_{y}^{2}<k_{0}^{2}} \vec{k}_{0} \times \overrightarrow{\tilde{E}}_{r}\left(k_{x}, k_{y}\right) \\
& \cdot e^{-j k_{z 0} z} e^{-j k_{x} x} e^{-j k_{y} y} d k_{x} d k_{y}
\end{aligned}
$$

where

$$
\overrightarrow{k_{0}}=k_{x} \hat{x}+k_{y} \hat{y}+k_{z 0} \hat{z} .
$$

The radiation loss can be evaluated by integrating the corresponding Poynting vector over an infinite plane $\left(z=z_{r}\right)$ that is above and parallel to the substrate surface [12]. The integral is given as

$$
P_{r}=\frac{1}{2} \operatorname{Re} \int_{-\infty}^{\infty} \int_{-\infty}^{\infty} \vec{E}_{r}\left(x, y, z_{r}\right) \times \vec{H}_{r}^{*}\left(x, y, z_{r}\right) \cdot \hat{z} d x d y
$$

Substituting (23) and (24) into (26), the radiation loss is found to be independent of the choice of $z_{r}$ and can be finally formulated in the spectral domain as

$$
\begin{aligned}
P_{r}= & \frac{1}{8 \pi^{2} \omega \mu_{0}} \iint_{k_{x}^{2}+k_{y}^{2}<k_{0}^{2}} k_{z 0}\left|\overrightarrow{\tilde{E}}_{r}\left(k_{x}, k_{y}\right)\right|^{2} d k_{x} d k_{y} \\
& -\frac{1}{8 \pi^{2} \omega \mu_{0}} \operatorname{Re} \iint_{k_{x}^{2}+k_{y}^{2}<k_{0}^{2}} \overrightarrow{\tilde{E}}_{r}\left(k_{x}, k_{y}\right) \\
& \cdot\left[\vec{k}_{0} \overrightarrow{\widetilde{E}}_{r}^{*}\left(k_{x}, k_{y}\right) \cdot \hat{z}\right] d k_{x} d k_{y} .
\end{aligned}
$$

The radiation patterns can be also computed from knowledge of the microstrip current densities. We can look upon the far 
fields as the Fourier transform of aperture fields located above the substrate. This leads to the expressions of the radiation patterns given as

$$
\begin{aligned}
E_{\phi}(\theta, \phi)=\hat{x} & \cdot \overrightarrow{\widetilde{E}}_{r}\left(k_{0} \sin \theta \cos \phi, k_{0} \sin \theta \sin \phi\right) \cos \theta \sin \phi \\
& -\hat{y} \cdot \overrightarrow{\widetilde{E}}_{r}\left(k_{0} \sin \theta \cos \phi, k_{0} \sin \theta \sin \phi\right) \cos \theta \cos \phi
\end{aligned}
$$

$$
\begin{aligned}
E_{\theta}(\theta, \phi)= & \hat{x} \cdot \overrightarrow{\tilde{E}}_{r}\left(k_{0} \sin \theta \cos \phi, k_{0} \sin \theta \sin \phi\right) \cos \phi \\
& +\hat{y} \cdot \overrightarrow{\tilde{E}}_{r}\left(k_{0} \sin \theta \cos \phi, k_{0} \sin \theta \sin \phi\right) \sin \phi .
\end{aligned}
$$

Surface waves propagating along the substrate can be investigated mathematically by the residue of the surface-wave poles in (21). When expressed in polar coordinates, the surface-wave electric field is written as

$$
\begin{array}{r}
\vec{E}_{\mathrm{Sw}}(\rho, \phi, z)=\frac{j}{2 \pi} \sum_{p} \lambda_{p} \int_{0}^{2 \pi} \overrightarrow{\widetilde{E}}_{\mathrm{sw}}\left(\lambda_{p}, \psi, z\right) \\
\cdot e^{-j \lambda_{p} \rho \cos (\psi-\phi)} d \psi
\end{array}
$$

where

$$
\begin{aligned}
\overrightarrow{\tilde{E}}_{\mathrm{sw}}\left(\lambda_{p}, \psi, z\right) & =\lim _{\lambda \rightarrow \lambda_{p}}\left(\lambda-\lambda_{p}\right) \overrightarrow{\widetilde{E}}(\lambda, \psi, z) \\
\lambda & =\sqrt{k_{x}^{2}+k_{y}^{2}} \\
\psi & =\tan ^{-1} \frac{k_{y}}{k_{x}} .
\end{aligned}
$$

Note that $\lambda_{p}$ represents the $p$ th surface-wave pole in the expression of the spectral-domain electric field. Considering the far fields, we use the method of steepest descent to obtain the following asymptotic form:

$$
\begin{gathered}
\vec{E}_{\mathrm{sw}}(\rho, \phi, z) \approx \frac{j}{\sqrt{2 \pi \rho}} \sum_{p} \sqrt{\lambda_{p}} \overrightarrow{\tilde{E}}_{\mathrm{sw}}\left(\lambda_{p}, \phi, z\right) e^{-j \lambda_{p} \rho}, \\
\text { as } \rho \rightarrow \infty .
\end{gathered}
$$

It is clearly seen in (34) that the surface waves in the far field behave as an outward-traveling cylindrical wave. The amplitude of the wave decreases as $\rho^{-(1 / 2)}$. The surface-wave far-field pattern can be further derived as

$$
\begin{aligned}
P_{\mathrm{sw}}(\phi)= & \frac{1}{2} \operatorname{Re} \int_{0}^{\infty} \vec{E}_{\mathrm{sw}}(\rho, \phi, z) \times \vec{H}_{\mathrm{sw}}^{*}(\rho, \phi, z) \cdot \hat{\rho} \rho d z \\
\approx & \frac{1}{4 \pi \omega \mu_{0}} \sum_{p} \lambda_{p}^{2} \int_{0}^{\infty}\left|\overrightarrow{\tilde{E}}_{\mathrm{sw}}\left(\lambda_{p}, \phi, z\right)\right|^{2} d z \\
& -\frac{1}{4 \pi \omega \mu_{0}} \sum_{p} \lambda_{p} \operatorname{Re} \int_{0}^{\infty} \overrightarrow{\tilde{E}}_{\mathrm{sw}}\left(\lambda_{p}, \phi, z\right) \\
& \cdot\left[\vec{k}_{\mathrm{sw}} \overrightarrow{\widetilde{E}}_{\mathrm{sw}}^{*}\left(\lambda_{p}, \phi, z\right) \cdot \hat{\rho}\right] d z
\end{aligned}
$$

where

$$
\vec{k}_{\mathrm{sw}}=\lambda_{p} \hat{\rho}+j \frac{\partial}{\partial z} \hat{z} .
$$

Note that the dependence with respect to $z$ in each component of $\overrightarrow{\widetilde{E}}_{\mathrm{Sw}}$ can be factored out as a harmonic function. This results
TABLE I

OPTIMIZED QUANTITIES OF THE SMALL-SIGNAL EQUIVALENT-CIRCUIT ELEMENTS

\begin{tabular}{c|c|c|c}
\hline$L_{g}$ & $L_{d}$ & $L_{s}$ & $R_{g}$ \\
\hline $1.687 \mathrm{nH}$ & $1.738 \mathrm{nH}$ & $0.212 \mathrm{nH}$ & $0.882 \Omega$ \\
\hline \hline$R_{d}$ & $R_{s}$ & $R_{g s}$ & $R_{g d}$ \\
\hline $0.36 \Omega$ & $0.313 \Omega$ & $35.7 \mathrm{M} \Omega$ & $93 \mathrm{M} \Omega$ \\
\hline \hline$R_{i}$ & $R_{d s}$ & $C_{g s}$ & $C_{g d}$ \\
\hline $9.35 \Omega$ & $590 \Omega$ & $0.536 \mathrm{pF}$ & $0.072 \mathrm{pF}$ \\
\hline$C_{d c}$ & $C_{d s}$ & $g_{m}$ & $\tau$ \\
\hline \hline $0.14 \mathrm{pF}$ & $0.48 \mathrm{pF}$ & $0.036 \mathrm{Sie}$ & $5.3 \mathrm{ps}$ \\
\hline
\end{tabular}
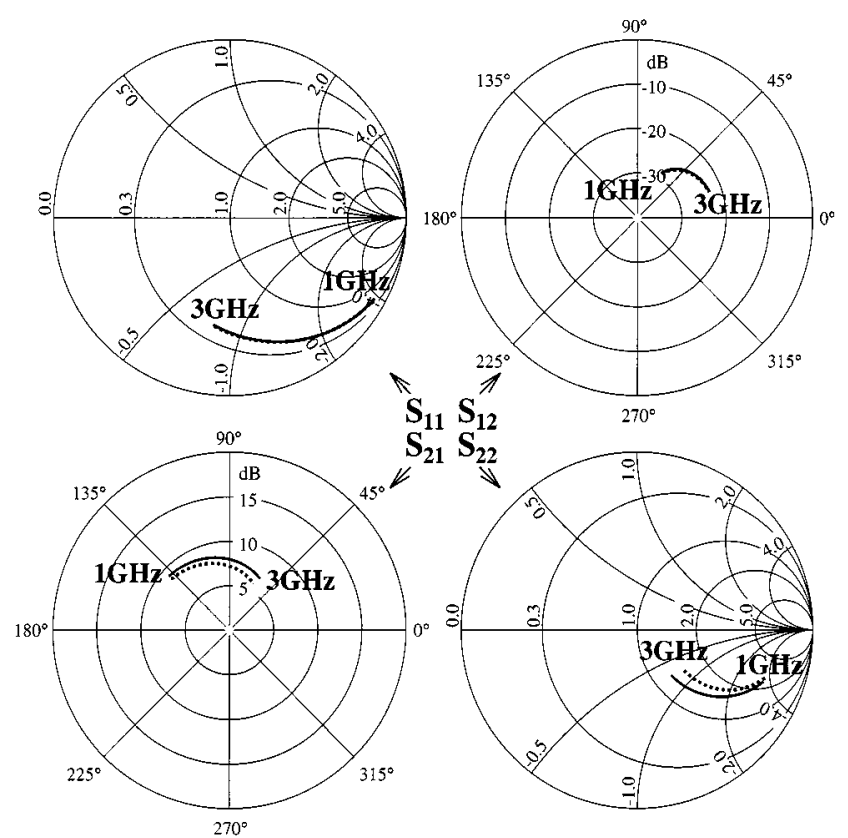

Fig. 4. Comparison of the scattering parameters calculated from the small-signal equivalent circuit in Fig. 2 with measurements. —: measurement. - - -: simulation.

in closed-form solutions for evaluating the integrals with respect to $z$ in the above equation. The surface-wave loss is finally calculated by

$$
P_{s}=\int_{0}^{2 \pi} P_{\mathrm{sw}}(\phi) d \phi
$$

\section{NUMERICAL RESUlTS AND DISCUSSION}

In the experiment, an NEC72084 GaAs MESFET was given dc bias at $V_{\mathrm{ds}}=3 \mathrm{~V}$ and $I_{d}=10 \mathrm{~mA}$ with the help of bias-T components. To ensure unconditional stability of the amplifier, a chip resistor $R_{\mathrm{st}}=48 \Omega$ was connected to the drain pad. The two-port scattering parameters for the MESFET connected with $50-\Omega$ microstrip lines and bias-T's at input and output were measured on an HP8510C with a frequency sweep from 1 to $3 \mathrm{GHz}$ and then deembedded to the reference planes, $x=x_{g}$ and $x=x_{d}$, where the via's centers were located. These deembedded scattering parameters can be converted into the MESFET's impedance parameters directly. However, for SPICE simulation applications, we developed an optimization procedure on HPEEsof's MDS to extract all the equivalent-circuit element values, as listed in Table I. It can be seen from Fig. 4 that, in the frequency range of $1-3 \mathrm{GHz}$, the scattering 

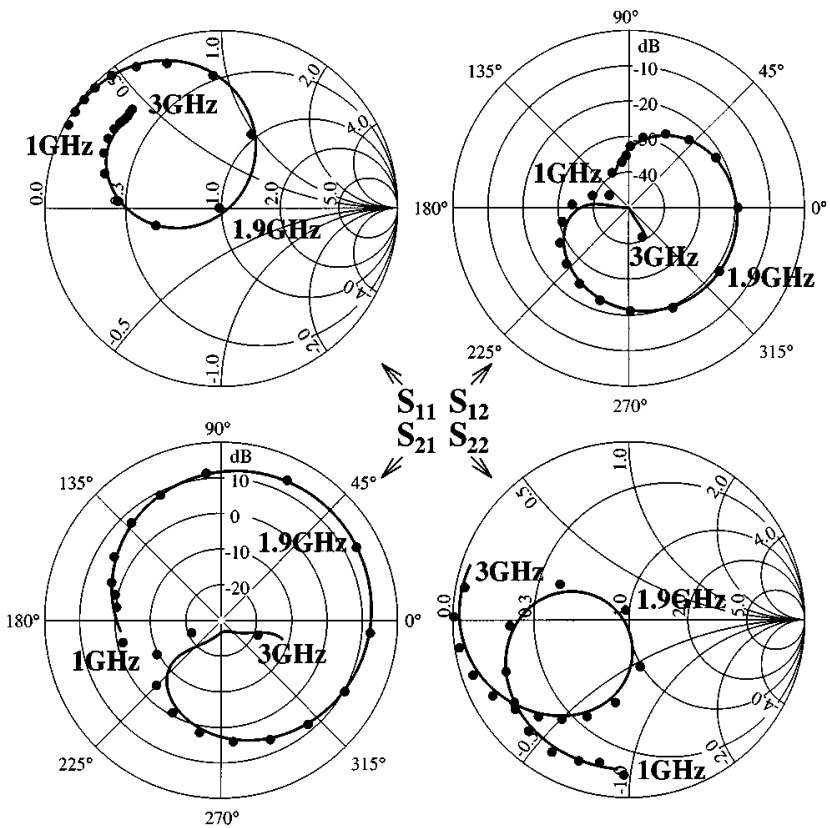

$\mathbf{S}_{21} \mathbf{S}_{22}$

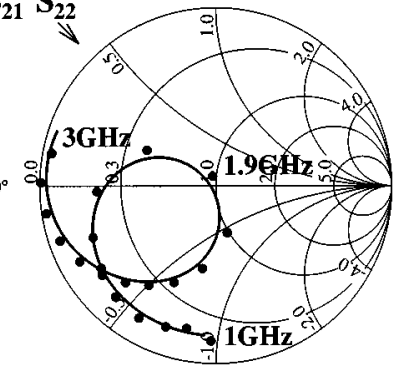

Fig. 5. Comparison of the simulated scattering parameters with measurements for the microstrip amplifier shown in Fig. 3. —: measurement. $\bullet \bullet \bullet$ : simulation.

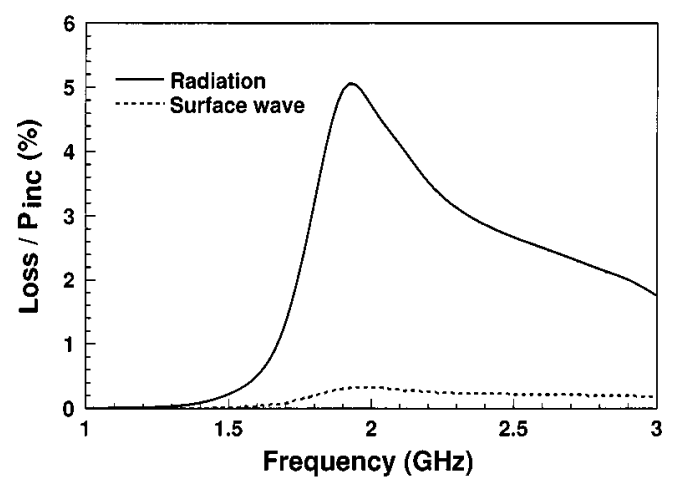

Fig. 6. Simulated radiation and surface-wave losses normalized by the incident power of the microstrip amplifier.

parameters calculated from the equivalent-circuit model agree quite well with the measured results. In the next step, we design the microstrip amplifier with a maximum gain at $1.9 \mathrm{GHz}$ for personal communication service (PCS) applications. The single-stub matching, as shown in Fig. 3, has been analyzed using the presented spectral-domain approach. As a result, the simulated scattering parameters for the microstrip amplifier are demonstrated in Fig. 5. Comparison with measurements also shows excellent agreement. One can observe that, at $1.9 \mathrm{GHz}$, the amplifier is well matched and reaches a maximum gain of $13.5 \mathrm{~dB}$.

The numerical results of the microstrip current densities in (20) are further used to evaluate the radiation and surface-wave losses. Fig. 6 shows both losses normalized by the incident power. It can be seen that maximum loss for both radiation and surface waves occurs at $1.9 \mathrm{GHz}$. This can be explained by the intensity of the microstrip current densities plotted in Fig. 7. At $1.9 \mathrm{GHz}$, the microstrip amplifier reaches its maximum gain and, meanwhile, has the most intensive current density for

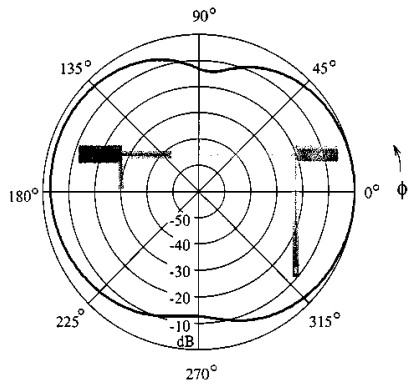

(a)

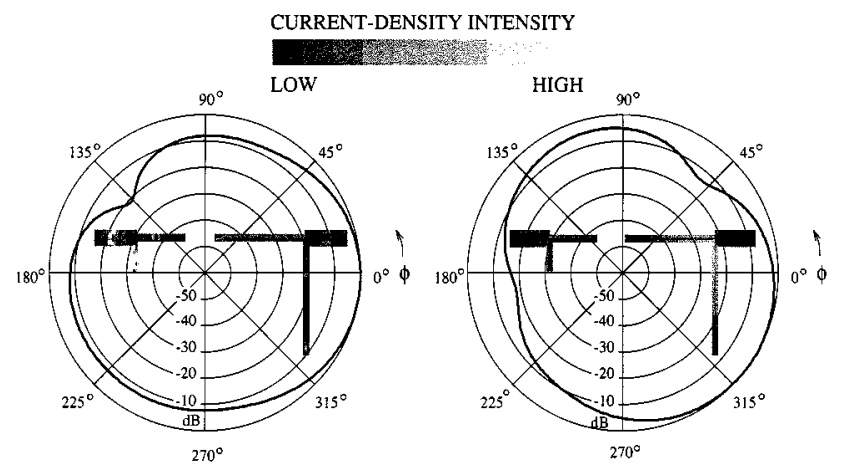

(b)

(c)

Fig. 7. Surface-wave power patterns generated by the current densities in the microstrip amplifier at the frequency of: (a) 1.9, (b) 1, and (c) $3 \mathrm{GHz}$.

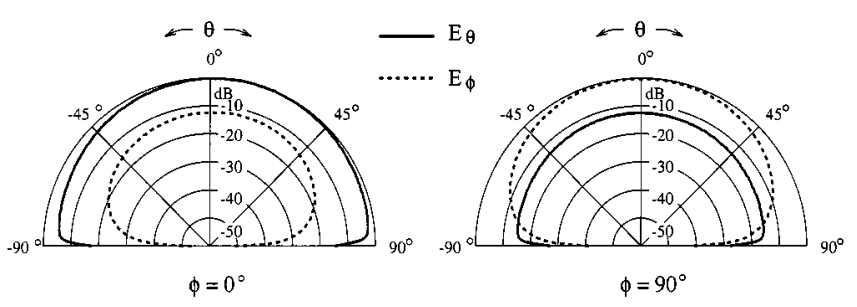

(a)

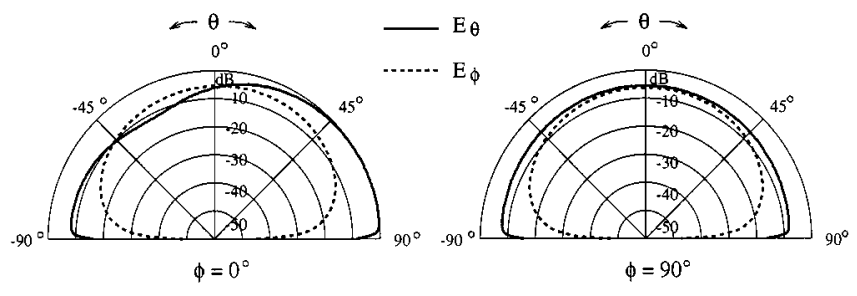

(b)
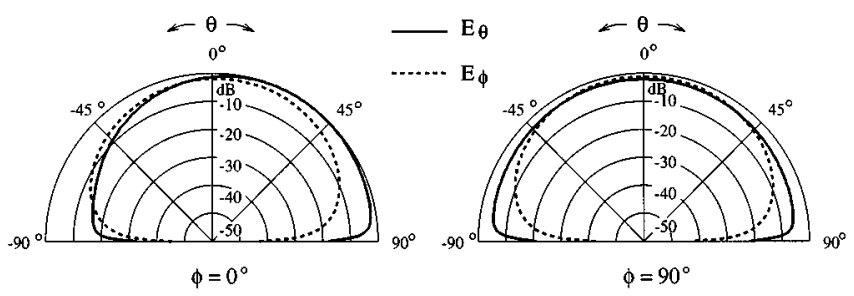

(c)

Fig. 8. $E$ - and $H$-plane radiation patterns for the microstrip amplifier operating at the frequency of: (a) 1.9 , (b) 1 , and (c) $3 \mathrm{GHz}$.

radiation. Fig. 7 also shows the surface-wave power patterns calculated from (35). Basically, the patterns point in the same direction as the microstrip currents propagate. It can be clearly found that, at $1.9 \mathrm{GHz}$, the $x$-directed current density dominates 


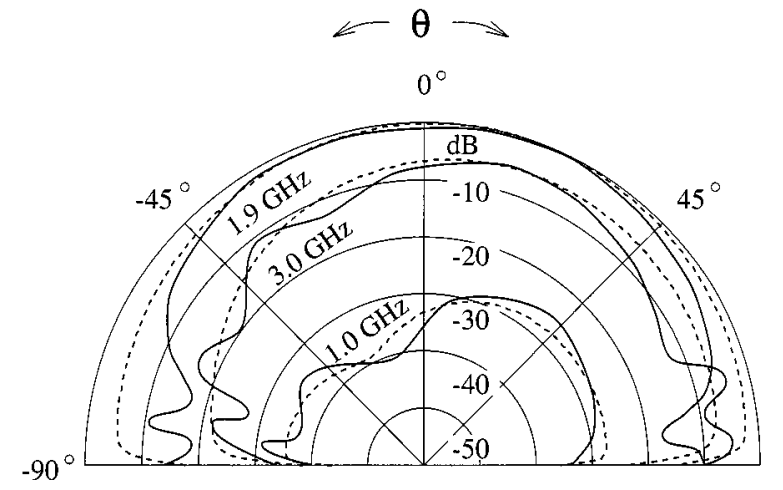

$90^{\circ}$

Fig. 9. Comparison of the simulated co-polarized far fields with measurements in the $E$-plane. - : measurement. - - -: simulation.

and the surface-wave pattern points at $\phi=0^{\circ}$. When the amplifier is not matched, say, at 1 and $3 \mathrm{GHz}$, the corresponding current densities become much less intensive in the $x$-direction. This makes the current densities in the microstrip stubs along the $y$-direction play an important role to tilt the surface-wave pattern. Similar explanations can be applied to the radiation patterns in Fig. 8, which are calculated from (28) and (29). Since the current density concentrates in the $x$-direction at $1.9 \mathrm{GHz}$, it is reasonable to find that the co-polarized field is larger by at least $10 \mathrm{~dB}$ than the cross-polarized field in both the $E$ - and $H$-plane. However, for the mismatch situations at 1 and $3 \mathrm{GHz}$, the $y$-directed current densities become relatively strong, raise the cross-polarized fields, and tilt the radiation patterns. To verify the theoretical results, we have measured the $E$-plane co-polarization patterns for the microstrip amplifier operating at 1, 1.9, and $3 \mathrm{GHz}$, respectively. Fig. 9 shows both calculated and measured patterns, which are normalized by the maximum co-polarized field intensity $E_{\theta}(\theta=0)$ at $1.9 \mathrm{GHz}$. As predicted, good agreement has been found.

\section{CONCLUSION}

A full-wave method has been presented to analyze radiation from a microstrip amplifier. A small-signal equivalent circuit of a GaAs MESFET has been incorporated in the spectral-domain EFIE formulation. The method of moments is applied to solve for the current densities distributed on the microstrip amplifier. These current densities cannot only determine the scattering parameters of the amplifier, but can also evaluate the losses due to radiated space and surface waves launched from the amplifier. Across the measured frequency range, the radiation loss dominates over the surface-wave loss. In addition, both losses increase with the intensity of the microstrip current densities. This explains why maximum losses have been observed when the microstrip amplifier is well matched.

\section{REFERENCES}

[1] S. C. Wu, H. Y. Yang, N. G. Alexopoulos, and I. Wolff, "A rigorous dispersive characterization of microstrip cross and T junctions," IEEE Trans. Microwave Theory Tech., vol. 38, pp. 1837-1844, Dec. 1990.
[2] T. Becks and I. Wolff, "Analysis of 3-D metallization structures by a full-wave spectral-domain technique," IEEE Trans. Microwave Theory Tech., vol. 40, pp. 2219-2227, Dec. 1992.

[3] T. S. Horng, W. E. McKinzie, and N. G. Alexopoulos, "Full-wave spectral-domain analysis of compensation of microstrip discontinuities using triangular subdomain functions," IEEE Trans. Microwave Theory Tech., vol. 40, pp. 2137-2147, Dec. 1992.

[4] M. J. Tsai, "Via-hole modeling for multi-layered microstrip circuits," M.S. thesis, Dept. Elect. Eng., Univ. California at Los Angeles, Los Angeles, CA, 1993.

[5] T. S. Horng, "A rigorous study of microstrip crossovers and their possible improvements," IEEE Trans. Microwave Theory Tech., vol. 42, pp. 1802-1806, Sept. 1994.

[6] J. R. Mosig, "Arbitrarily shaped microstrip structures and their analysis with a mixed potential integral equation," IEEE Trans. Microwave Theory Tech., vol. 36, pp. 314-323, Feb. 1988.

[7] K. A. Michalski and D. Zheng, "Analysis of microstrip resonators of arbitrary shape," IEEE Trans. Microwave Theory Tech., vol. 40, pp. 112-119, Jan. 1992.

[8] M. J. Tsai, C. Chen, N. G. Alexopoulos, and T. S. Horng, "Multiple arbitrary shape via-hole and air-bridge transitions in multilayered structures," IEEE Trans. Microwave Theory Tech., vol. 44, pp. 2504-2511, Dec. 1996.

[9] W. Sui, D. A. Christensen, and C. H. Durney, "Extending the two-dimensional FDTD method to hybrid electromagnetic systems with active and passive lumped elements," IEEE Trans. Microwave Theory Tech., vol. 40, pp. 724-730, Apr. 1992.

[10] C.-N. Kuo, V. A. Thomas, S. T. Chew, B. Houshmand, and T. Itoh, "Small signal analysis of active circuits using FDTD algorithm," IEEE Microwave Guided Wave Lett., vol. 5, pp. 216-218, July 1995.

[11] C.-N. Kuo, B. Houshmand, and T. Itoh, "Full-wave analysis of packaged microwave circuits with active and nonlinear devices: An FDTD approach," IEEE Trans. Microwave Theory Tech., vol. 45, pp. 819-826, May 1997.

[12] T. S. Horng, S. C. Wu, H. Y. Yang, and N. G. Alexopoulos, "A generalized method for distinguishing between radiation and surface-wave losses in microstrip discontinuities," IEEE Trans. Microwave Theory Tech., vol. 38, pp. 1800-1807, Dec. 1990.

[13] T. S. Horng and S. M. Wu, "Full-wave analysis of radiation from a microstrip amplifier," in IEEE MTT-S Int. Microwave Symp. Dig., 1999, pp. $1677-1680$

[14] T. S. Horng, "A rigorous study of wire-bonding and via-hole effects on GaAs field effect transistors," in IEEE MTT-S Int. Microwave Symp. Dig., 1995, pp. 785-788.

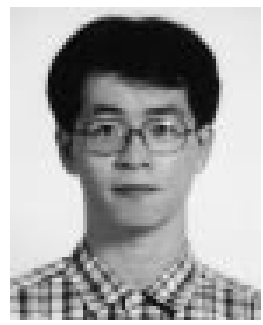

Tzyy-Sheng Horng (S'88-M'92) was born in Taichung, Taiwan, R.O.C., on December 7, 1963. He received the B.S.E.E. degree from the National Taiwan University, Taipei, Taiwan, R.O.C., in 1985, and the M.S.E.E. and Ph.D. degrees from the University of California at Los Angeles, in 1990 and 1992, respectively.

$\mathrm{He}$ is currently an Associate Professor with the Department of Electrical Engineering, National Sun Yat-Sen University, Kaohsiung, Taiwan, R.O.C. His research interests are in the area of RF and microwave integrated circuits and packages.

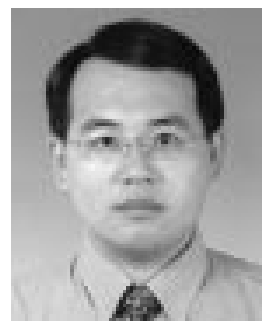

Sung-Mao Wu was born in Kaohsiung, Taiwan, R.O.C., on January 1, 1971. He received the B.S.E.E. degree from the Fu Jen Catholic University, Taipei, Taiwan, R.O.C., in 1994, the M.S.E.E. degree from the Yunlin University of Science and Technology, Yunlin, Taiwan, R.O.C., in 1996, and is currently working toward the Ph.D. degree in electrical engineering at the National Sun Yat-Sen University, Kaohsiung, Taiwan, R.O.C. 